\title{
Evaluation of a New Method and Diagnostic Test in Semen Analysis
}

\author{
Petra Zrimšek \\ Clinic for Reproduction and Horses, \\ Veterinary Faculty, \\ University of Ljubljana, \\ Slovenia
}

\section{Introduction}

Sperm concentration is an important parameter affecting fertility. Animal species of agricultural interest are mainly produced by artificial insemination (AI) which contributes highly to the development of worldwide swine production, making the impact of the male in reproductive efficiency of the pig herds more crucial (Jounala et al., 1998).

The efficiency of AI (fertility rate and prolificacy) is directly dependent on the quality of semen doses and on the number of spermatozoa used for insemination (Camus et al., 2011). In commercial farms, routine examination of boar semen is performed aiming to predict the male's fertility. Evaluation of concentration is crucial to adapt dilution rate and to optimize sperm concentration which will directly impact fertility performance. In the first part of a present chapter we address the basic concepts of a method comparison study and present an example of a method comparison experiment concerning determination of sperm concentration.

Various laboratory methods techniques are used to evaluate sperm quality, such as sperm concentration, motility, viability, and morphology. However, there is no single semen assay that provides complete information about semen quality (Holt \& Medrano, 1997; Johnson et al., 2000; Liu \& Baker, 2002). Studies in domestic animals showed that these semen characteristics were often not significantly correlated to fertility, while the most valid assessment of boar semen quality is to obtain viable pregnancies and normal offspring following AI (Tsakmakidis et al., 2010). Since fertilization is a complex process involving a huge number of events, fertility research must not only device more predictive laboratory tests, but also properly combine different assays aiming to predict male fertilizing ability, as spermatozoa should satisfy many requirements for successful fertilization (QuinteroMoreno et al., 2004). Assessment of metabolic status of spermatozoa could provide a useful tool for evaluation of semen quality, because sufficient metabolism for energy production is one of the several attributes that a sperm must posses to fertilize an oocyte. In the second part of this chapter developing and diagnostic evaluation of a spectrophotometric application of the resazurin reduction assay will be presented.

Learning objectives of a chapter are to:

- Investigate repeatability in continuous data 
- $\quad$ Perform method agreement

- Construct Bland-Altman plots

- Explain limits of agreement between two methods

- Chose an appropriate regression analysis used in the interpretation of comparing data

- Define the diagnostic parameters: specificity, sensitivity, accuracy, predictive values of a test

- $\quad$ Recognize the validity and usefulness of the test

- Evaluate the performance of a diagnostic test using ROC (receiver operating characteristic) analysis

- Construct and compare ROC curves

- Determine optimal cut-off point for a test

- Explain the developing of a new method in semen evaluation

\section{Method agreement for determining sperm concentration}

Semen samples, which often contain a variety of cells (immature germ cells, blood cells, epithelial cells, and cellular debris) in addition to spermatozoa, differ markedly from blood samples because of their heterogeneity. There is also no specific standard available for sperm cells of each species. It is therefore important to compare a new, more appropriate or additional method to a conventional one. The counting chamber technique for estimating sperm count appears to be adequate because of its simplicity, low cost and reproducibility. However, photometers are widely used routinely for determining sperm concentration by many AI organisations, for bulls and boars as well as other species (Woelders, 1991). They need to be evaluated before use, because accurate concentration measurement is the first and crucial step of the semen preparation process for production of semen doses (Camus et al., 2011). Correct assessment of sperm concentration is essential to ensure that the number of sperm per insemination dose meets requirements and that the maximal number of doses can be produced per ejaculate.

The increasing use of $\mathrm{AI}$ in swine emphasizes the need for the distribution of good quality sperm by the AI centres (Vyt et al., 2004). Boar sperm quality is routinely assessed by measuring concentration, morphology and motility of spermatozoa (Johnson et al., 2000). Determination of sperm concentration is essential in evaluating fertility, whether in vivo or in vitro. However, there is no agreed method for use as a standard. Knuth et al. (1989) showed that introduction of an unevaluated laboratory method, without appropriate quality control, can cause a bias in semen analysis. However, the methodology of semen evaluation is complex, and standardization is difficult (Brazil et al., 2004). For example, the first large scale, nation-wide proficiency testing program for clinical andrology laboratories in the United States reported that the inter-laboratory coefficient of variation for manual sperm concentration determination was $80 \%$, with a range for a single semen specimen of $3-492 x$ $10^{6} / \mathrm{ml}$ (Keel et al., 2000). The accuracy, reliability and repeatability of different instruments that evaluate sperm concentration of raw semen have already been compared in several previous studies (Christensen et al., 2004; Hansen et al., 2006; Prathalingam et al., 2006; Anzar et al., 2009; Camus et al., 2011). Variation in the results from different laboratories could be due to the lack of standardisation of methods between laboratories (Maatson, 1995).

The reason for comparing methods is often that a quicker, more convenient and more economical adaptation has been made to an existing method. Studies comparing a new 
method with an established method are performed to assess whether the new measurements are comparable with existing ones (Jensen \& Kjelgaard-Hansen, 2006).

\subsection{Precision of the evaluated methods}

It is necessary to establish that a method is repeatable before comparing two measurements for reproducibility (Petrie \& Watson, 1999). Repeatability of boar semen concentration assessment depends on instruments and procedures, for example CV for instruments FACS, HEMO, Photo C254, SpermVision, UltiMate and SP-100 were 2.7, 7.1, 10.4, 8.1, 5.4 and 3.1\%, respectively (Hansen et al., 2006). Imade et al. (1993) reported similar overall precision (5.9\%) for the Makler chamber, whereas CV for sperm counts in sperm suspensions can be higher, for example 18.6\% (Christensen et al., 2005) or even 26.3\% (Mahmoud et al, 1997). It is generally admitted that intra-observer CVs are often greater than $10 \%$. Although guidelines for standardizing the procedure have been proposed, relatively important degrees of intra- and inter- technician or inter-laboratory variability have been reported. In the external quality assessment (EQA) reported by Neuwinger and coworkers (1990), which involved 10 experienced German laboratories in the evaluation of 8 sperm samples, the mean CV was $37.5 \%$. From the data of the external quality control obtained under the British Fertility Society and reported by Matson (1995), the calculated inter-individual CV for sperm concentration was $64.7 \%$ for 24 semen samples collected by technicians from 20 laboratories.

\subsection{Method agreement}

According to the literature, a very common way of investigating method agreement is to perform a paired t-test or to calculate a correlation coefficient to provide a measure of agreement. However, in this instance, neither method is appropriate for the following reasons (Petrie \& Watson, 1999). The paired t-test tests the null hypothesis that the difference is zero. If the differences between pairs are large - indicating that the methods do not agree - but are evenly scattered around zero, then the result is non-significant. We can only conclude that there is no bias, not that the methods agree. Correlation is a statistical method used to quantify any association between two continuous variables (Ma \& Smith, 2003). The correlation coefficient provides a measure of the linear association between the measurements obtained by the two methods. It provides an indication of how close the observations in the scatter diagram are to a straight line. $\mathrm{R}$ measures the strength of a relation between two variables, not the agreement between them (Bland and Altman, 1999). For example, the Pearson correlation coefficient gives no information of value in method comparison studies, because $\mathrm{R}$ can be highly significant even when there is an obvious bias between the two methods. It measures the strength of association, rather than agreement, although in the literature it has been used in many studies, such as comparison between different methods to determine sperm concentration (Prathalingam et al., 2006). $\mathrm{R}$ was also used to evaluate agreement between assessments within lab technician in sperm analysis (Christensen et al., 2005). In order to assess agreement, it is necessary to know how close the points are to the line of equality, i.e. the $45^{\circ}$ line (Petrie \& Watson, 1999). Therefore, in the study of Sokol et al. (2000), comparison of two methods for measuring sperm concentration using only Wilcoxon signed rank test and F-test, appears to be insufficient.

Scatter plots and absolute and relative bias plots give the best overview of comparisons of data (Twormey, 2004; Twormey, 2005). Absolute bias plots are also called Bland and Altman 
plots, usually used for method comparison (Bland and Altman, 1999). In absolute bias plots, the biases are plotted against their average value for each sample. The mean of these differences $(\bar{d})$ is an estimate of the average bias of one method relative to that of the other. If this value is zero, then the two measurements agree on average. However, this does not imply that they agree for each individual measurement.

In order to assess how well the paired measurements agreed with each other, limits of agreement have to be determined. The upper and lower limits of agreement are calculated as

$$
\bar{d} \pm 2 \mathrm{~s}_{\text {difff }}
$$

where $\bar{d}$ is the mean of differences for all the samples (average bias) and $s_{\text {diff }}$ is the standard deviation of the differences; $2 \mathrm{~s}_{\text {diff }}$ is also referred to as the British Standard Institution repeatability (or, reproducibility, as appropriate) coefficient and indicates the maximum difference likely to occur between two measurements. This coefficient is the value below which the bias between paired results may be expected to lie (Petrie \& Watson, 1999).

We performed method agreement between two clinical laboratory methods for determining boar sperm concentration using the statistical programme Analyse-it, General + Clinical Laboratory statistics, version 1.71, where linear regression, Deming regression and Passing Bablok regression can be applied in the evaluation. We chose Deming regression, because it is appropriate for describing the relationship between two variables, both measured with error. In the case of observed increasing imprecision, i.e. where a proportional bias between methods is detected, the Passing Bablock regression procedure is more accurate than Deming's method. When the assumption that the independent variable is determined without error is satisfied, linear regression should be used to describe the agreement between two methods (Jones \& Payne, 1997). The intercept is calculated, as in conventional least squares regression, as the mean of $y$ minus the product of the slope and the mean of $x$. The standard error (SE) of the intercept defines how much the line might vary in the $y$ direction, and SE of the slope defines how much the line might pivot about the central point through the means of $x$ and $y$. Thus, SEs allow calculation of the confidence intervals of the slope and the intercept (Jones \& Payne, 1997).

\subsection{Experiment: Agreement between two methods of sperm concentration measurement}

In the present study we compared two clinical laboratory methods for determining boar sperm concentration, the Makler chamber and the photometer (Photometer SDM5, Minitüb, Germany) (Mrkun et al., 2007). Prior to method comparison, precision of each method was assessed. Scatter plots with fitted regression line, and absolute and relative bias plots were used to get the best overview of comparative data (Twormey, 2004; Twormey, 2005). Deming regression was applied to describe the relationship between variables both measured with error by proposing that the sum of the squares of the deviations from a line should be minimised in both the $\mathrm{x}$ and the $\mathrm{y}$ directions at the same time, thus taking account of the analytical imprecision of each method (Jones \& Payne, 1997). The purpose of this study was to compare the two methods and to assess method agreement together with the appropriate regression analysis used in the interpretation of the data. 


\subsubsection{Semen samples}

Twenty-three semen samples were obtained from eight 12 to 24 month old boars of various breeds. Each semen sample was collected with gloved hand using a clean semen collecting flask that filters out gel, dust and bristles, while the boar mounted a dummy sow. Semen samples were diluted 1:2 with BTS semen extender (Beltsville Thawing Solution, Truadeco, Netherlands) and delivered to the laboratory.

\subsubsection{Counting with the Makler chamber}

Immediately before each semen aliquot was analysed, the entire semen specimen was vortexed. To render the spermatozoa immotile and to prepare the semen samples for the Makler chamber (Sefi Medical Instruments, Israel), semen samples were diluted 1:2 with distilled water. 6 parallel dilutions of each semen sample were prepared and the average of the measurements on each used as the representative value.

Following dilution, sperm suspensions were again vortexed and an aliquot of $5 \mu \mathrm{l}$ was loaded into the Makler chamber. The next step was to assess whether sperm were evenly distributed or whether there were movements in the fluid in the counting chamber. If either of these problems was observed; the chamber was cleaned and refilled. The fields were chosen according to a prescribed pattern: 10 fields spaced left to right and 10 fields spaced top to bottom. Chosen fields formed a plus sign centred in the middle of the chamber, excluding the areas 2-3 $\mathrm{mm}$ from the chamber edges. Only recognizable spermatozoa, including lost heads, were counted, while other cells and lost tails were ignored. The concentration in the original semen sample was calculated from the total number of sperm in the counting area.

\subsubsection{Counting with a photometer}

Sperm concentration was determined by measuring the sample opacity, as the percentage transmittance of light through a sample, using a photometer (Photometer SDM5, MiniTüb, Germany). Boar ejaculates are normally too opaque, so a small semen sample was diluted with an isotonic solution before measuring. A blank tube was loaded with $3.5 \mathrm{ml} 0.9 \% \mathrm{NaCl}$ and a sample tube with $70 \mu \mathrm{l}$ semen sample added to $0.9 \% \mathrm{NaCl}$. Sperm concentration was determined from a previous calibration of the spectrophotometer, performed by the manufacturer (Photometer SDM5, MiniTüb, Germany). Six measurements were made for each semen dilution.

\subsubsection{Precision of the evaluated methods}

The precision of each method was determined by making 6 measurements of each of 23 semen samples. Coefficients of variation (CV) were calculated for each method and scatter graphs of $\mathrm{CV}$ versus average sperm count for each semen sample were constructed. In our study CVs were calculated to be $6.6 \pm 3.5 \%$ and $1.6 \pm 0.6 \%$ for Makler chamber and photometer, respectively. Both methods yielded acceptable precision (Christensen et al., 2005), although the precision of the Makler chamber was significantly poorer.

In a diagram of the CV plotted against the average for each sperm concentration, the scatter of the points is random for the photometer (Fig.1). In contrast, for the Makler chamber, the size of $\mathrm{CV}$ is related to the size of the sperm concentration, shown by the higher CVs for lower sperm counts (Fig.2). 


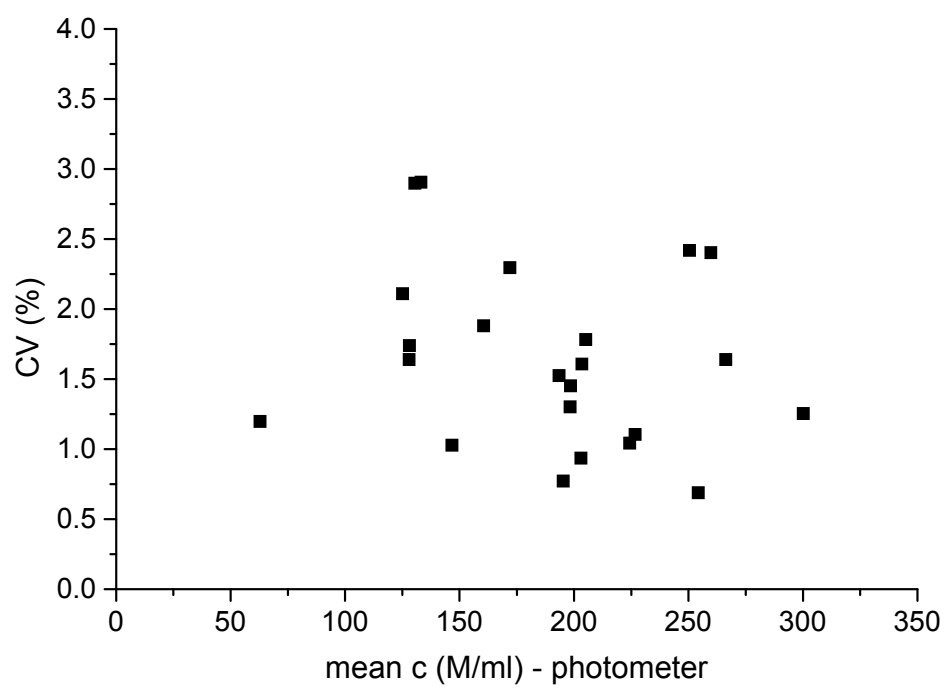

Fig. 1. Coefficient of variation $(\mathrm{CV})$ versus mean sperm count for the photometer method Mean counts were calculated as the average of six parallel counts for each sample

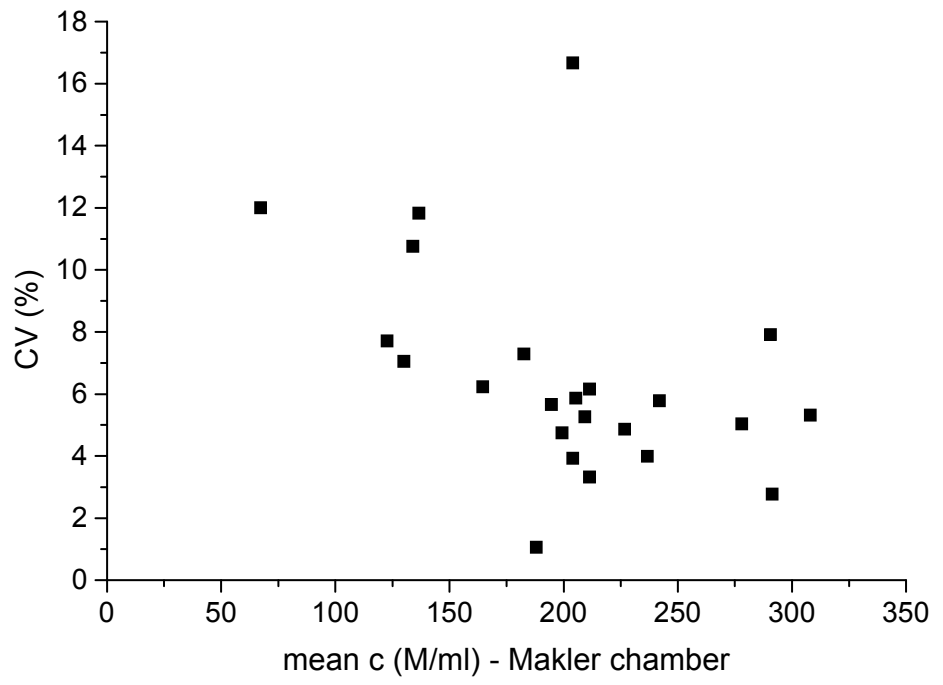

Fig. 2. Coefficient of variation (CV) versus mean sperm count determined by the Makler chamber method

Mean counts were calculated as the average of six parallel counts for each sample 


\subsubsection{Method agreement between Mackler chamber and photometer}

We were interested in assessing the similarity between sperm counts measured with Makler chamber and photometer, so we compared pairs of measurements. For this purpose, we calculated the differences between pairs of measurements of sperm counts - by Makler chamber and photometer - obtained by each method for each sperm sample.

The mean percentage bias between methods was $-0.6 \pm 6.9 \%$. The scatter of the points lies in the interval -15 to $15 \%$ (Fig. 3), which is in the range of satisfactory between-run reproducibility of the assay. From the absolute bias plot (Fig. 4) it is also evident that the scatter is random, indicating that the size of the difference between sperm counts obtained by two methods is not related to the size of the counts. Thus, no proportional bias has been detected. Average absolute bias was close to zero $(-1.092 \pm 15.237 \mathrm{M} / \mathrm{ml})$. Sperm counts obtained with Makler chamber and photometer agree; $90 \%$ of the differences lie within the limits of agreement (Fig. 4), confirming that the level of agreement between the methods was satisfactory. Therefore, measurements of sperm concentration with photometer and counting chamber techniques are equally appropriate for estimating sperm counts.

Using scatter diagrams with regression lines fitted, we established that the paired measurements, sperm counts obtained with Makler chamber and with photometer, were close to the line of equality. Deming regression was used to solve the problem of describing the relationship between sperm counting with methods, both measured with error. Deming's method gives only a single regression line, whether $\mathrm{x}$ or $\mathrm{y}$ is used as the "independent variable" (Fig. 5).

The estimated intercept for the regression line, $4.7069 \mathrm{M} / \mathrm{ml}$, does not differ much from zero. The estimated regression equation indicates that the points are close to the line of equality, i.e. the $45^{\circ}$ line and SE of the slope (0.0600) indicates that there is almost no pivoting of the line about central point through the means of $x$ and $y$.

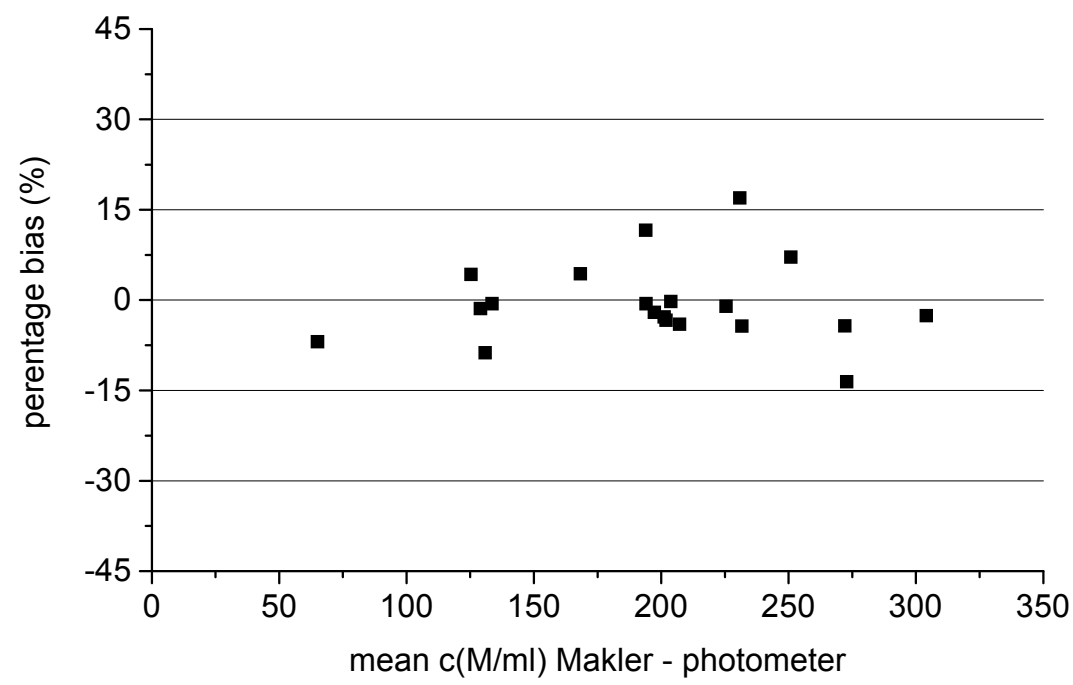

Fig. 3. Relative bias plot of sperm concentration obtained by Makler chamber versus concentration obtained by photometer 


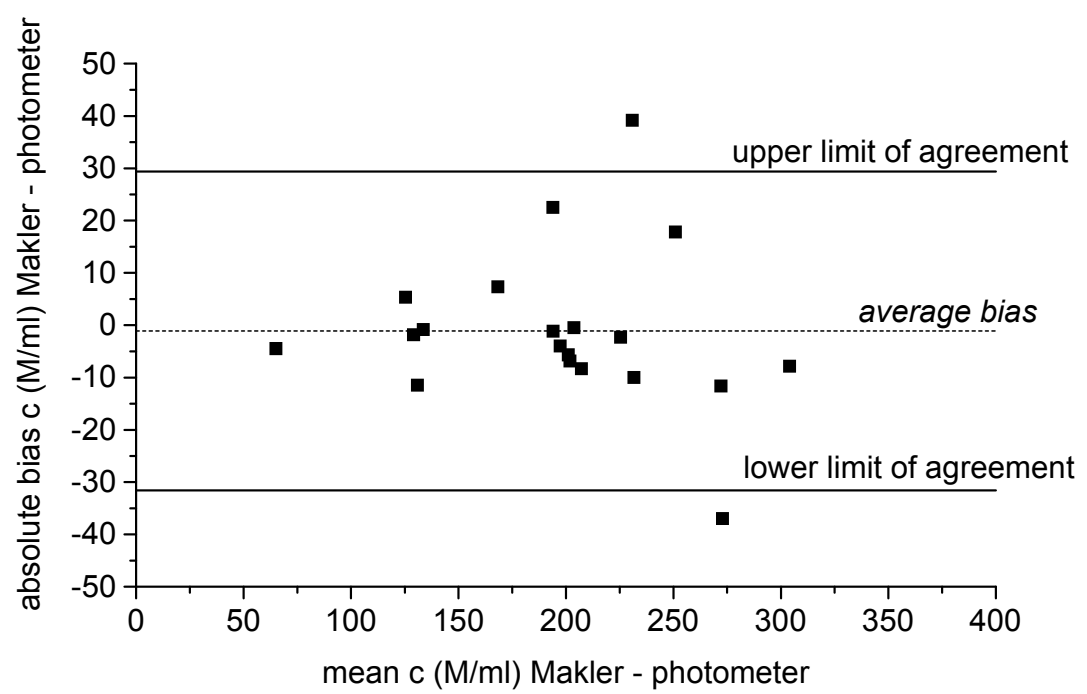

Fig. 4. Absolute bias plot of sperm concentrations obtained by Makler chamber versus concentrations obtained by photometer showing average bias and limits of agreement

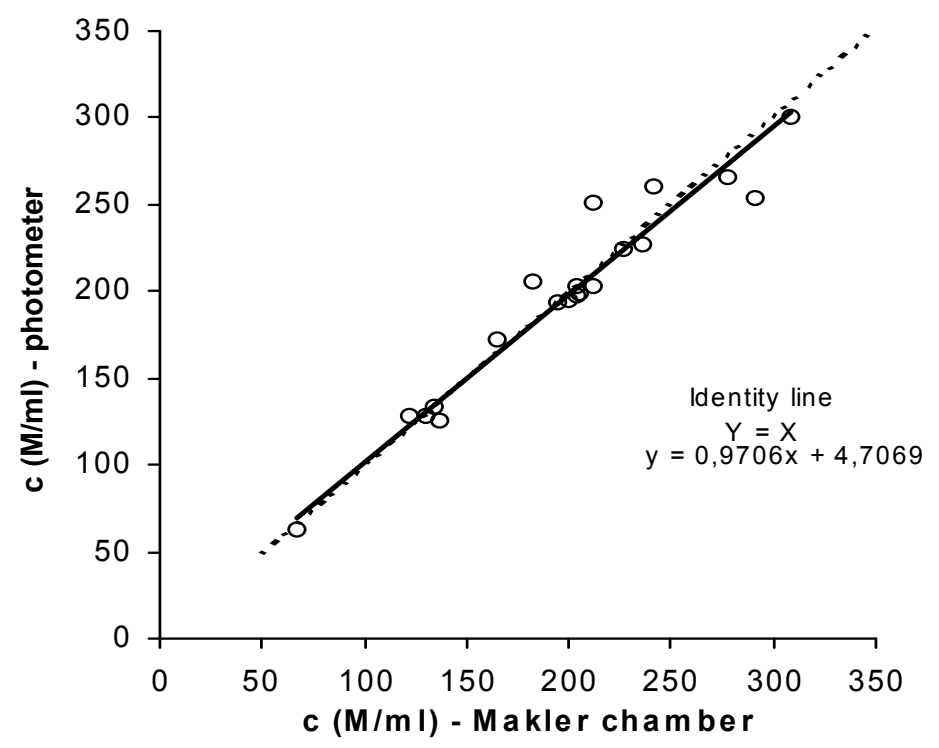

Fig. 5. Scatter diagram of sperm concentration obtained by photometer versus sperm concentration obtained with Makler chamber, with Deming regression line fitted - . - : line of equality $(\mathrm{Y}=\mathrm{X})$

: Deming regression line:

$\mathrm{c}($ photometer $)=4.7069+0.9706 \times \mathrm{c}($ Makler chamber $)$ 


\section{Development and diagnostic evaluation of spectrophotometric application of the resazurin reduction assay to evaluate boar sperm quality}

There are several attributes that a sperm must posses to fertilize an oocyte, including motility, normal morphology, sufficient metabolism for energy production, and membrane integrity. Although various analytical techniques have been developed to evaluate sperm quality, including sperm concentration, motility, viability and morphology, there is no single method that provides complete information about semen quality (Holt \& Medrano, 1997; Johnson et al., 2000). Due to the complexity of the fertilization process, single tests are not able to predict fertility. Instead, a set of semen tests has to be selected with high relevance for important sperm traits and low redundancy of assay results (Petrunkina et al., 2007). Moreover, particularly in pig industry, the choice of semen test has considered cost effectiveness. Routine testing of fresh boar sperm predominantly aims to identify subfertile boar ejaculates. In number of countries, liquid preserved boar semen is used after several days of in vitro storage. It's well known that boars differ in their capacity to maintain sperm function during preservation in vitro. These differences can only be partially visualized by standard sperm parameters, such as loss of motility and membrane integrity (Waberski et al., 2011). However, the battery of diagnostic methods used by the industry is as yet restricted (Tejerina et al., 2008). A reliable, simple, cost effective and rapid method of assessing the quality of boar spermatozoa would be of benefit to livestock producers and veterinary practitioners (Dart et al., 1994). Reproductive performance depends on metabolic processes; therefore assessment of metabolic status of spermatozoa could provide valuable information for predicting sperm fertilizing capacity. The resazurin reduction assay, one of the methods for evaluating the metabolic status of spermatozoa, depends on the ability of metabolically active spermatozoa to reduce the resazurin redox dye to resorufin. Dehydrogenase activity of spermatozoa is manifested as a visual colour change from blue (resazurin) to pink (resorufin) and further to white (dihydroresorufin) (Glass et al., 1991; Fuse et al., 1993; Reddy Venkata Rami et al., 1997). The resazurin reduction assay using visual detection of colour change is quite subjective and varies between evaluators (Wang et al., 1998). The colour change of resazurin is usually matched with a colour chart, consisting of a spectrum of colours from blue to pink, varying between investigators. The possibility of human error therefore, has led to the spectrophotometric modification of the resazurin reduction test. It has been mostly used for the evaluation of human semen (Mahmoud et al., 1994, Rahman \& Kula, 1997; Zalata et al., 1998; Reddy Venkata Rami \& Bordekar; 1999) but, to our knowledge, in veterinary medicine only for evaluating ram (Wang et al., 1998) and boar semen quality (Zrimšek et al., 2004). The visual assay has been used for evaluating stallion (Carter \& Ericsson, 1998), bull (Dart et al., 1994), sheep (Cooper et al., 1996; Martin et al., 1999) and boar (Mesta et al., 1995) semen. Spectrophotometric measurement of resazurin reduction provides a quantitative and objective method.

The aim of the present study was to develop and evaluate diagnostically the spectrophotometric application of the resazurin reduction test for evaluating boar sperm quality (Zrimšek et al, 2004; Zrimšek et al., 2006). Following Zalata et al. (1998), who developed a spectrophotometric method of resazurin reduction to evaluate human semen we extracted the developed colour after the assay with boar semen with butanol and measured the absorbance in the clear upper layer of butanol, eliminating the problem of sample turbidity. The optimisation and developing of the test included several steps as follows:

- determination of specific absorbance wavelength, used for analysis on the basis of absorbance spectra of resazurin and resorufin 
- $\quad$ optimisation of the test procedure

- determination of repeatability of the assay

- correlations between resazurin reduction assay and various semen parameters; Spearman rank correlation analysis

- relationship between resazurin reduction and concentration of motile spermatozoa and sperm index; linear regression analysis

- $\quad$ statistical comparison of the results obtained between the groups of satisfactory and unsatisfactory semen; Mann-Whitney U-test

- diagnostic evaluation of the assay; ROC analysis

- $\quad$ stability of butanol extracts in terms of $\mathrm{A}_{610}$; measuring agreement

In this study, receiver operating characteristics (ROC) was used to determine the optimal cut-off value and diagnostic accuracy of the resazurin reduction assay. A complete picture of test accuracy is presented by the ROC plot, which provides a view of the whole spectrum of sensitivities and specificities as functions of selected cut-off values (Greiner et al., 2000). A global summary statistic of the diagnostic accuracy of the assay was quantified by the area under the ROC curve. Likelihood ratios were used to revise the probability of the semen status in individual samples (Greiner et al., 1995).

\subsection{Development of resazurin reduction assay}

\subsubsection{Semen samples and analysis}

Forty-one semen samples from eight 12-24-month-old boars of various breeds were included in the study. Semen was collected with a glove hand using a clean semen collecting flask that filters out gel, dust and bristles, while the boar mounted a dummy sow. Semen was kept at the temperature collected and analyzed within $1 \mathrm{~h}$. Sperm concentration and motility characteristics were determined by computer-assisted semen analysis (Hamilton Thorne IVOS 10.2; Hamilton Thorne Research, MA, USA) with a Makler counting chamber (Sefi Medical Instruments, Haifa, Israel). Sperm morphology was examined on Giemsa-stained samples (Hafez, 1993). Sperm index (SI) was calculated by multiplying sperm concentration by the square root of percentage sperm motility multiplied by the percentage of normal sperm morphology (Mahmoud et al., 1994). Combining concentration, motility and morphology in sperm index allows the concentration of active spermatozoa to be determined, and may provide a better means of evaluating semen quality than assessing the characteristics, mentioned above, independently.

\subsubsection{Determination of specific absorbance wavelengths of resazurin and resorufin}

Before developing the assay, specific absorbance wavelengths of resazurin and resorufin were determined. Ten $\mu 11.8 \mathrm{mM}$ resazurin (Sigma, Steinheim, Germany) in physiological saline was added to $1 \mathrm{ml}$ of $1: 2$ dilution of semen sample in BTS and incubated at $37^{\circ} \mathrm{C}$ in a water bath. After the semen sample completely turned to pink, the developed dye (resorufin) was extracted from the solution by adding n-butyl alcohol (Sigma, Germany) and fast vortexing. The control sample (blue colour solution) was prepared by adding butanol immediately after the resazurin. After centrifugation, the blue (resazurin) and pink (resorufin) solutions were separated from the clear upper layers of butyl alcohol and were scanned in the range from 400 to $850 \mathrm{~nm}$, using a scanning spectrophotometer (UV/VIS Spectrometer Lambda 12, Perkin Elmer). Resazurin exhibits an absorption peak at $610 \mathrm{~nm}$, 
while that of resorufin is at $575 \mathrm{~nm}$ (Fig. 6). There was minimal overlapping between absorption peaks of resazurin and resorufin at $610 \mathrm{~nm}$; therefore the absorbance at $610 \mathrm{~nm}$ was used in further analysis.

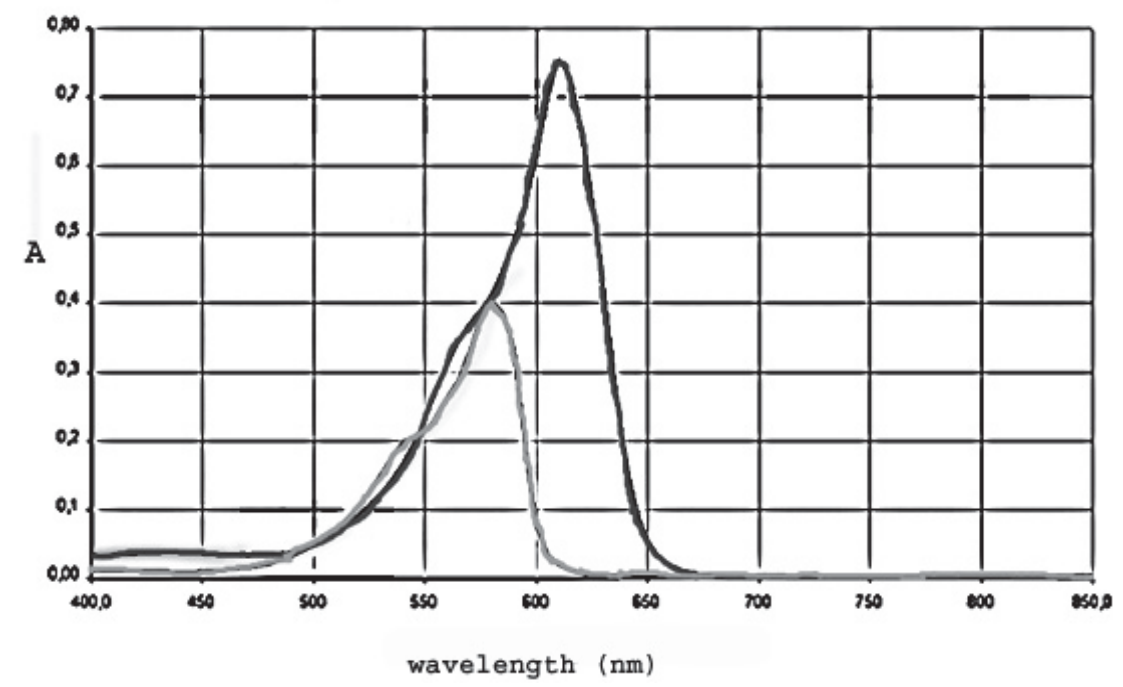

Fig. 6. Specific absorbance wavelengths of resazurin (-) and resorufin (-)

\subsubsection{Resazurin reduction assay and correlation with semen parameters}

The resazurin reduction assay was performed within $1 \mathrm{~h}$ after semen collection. Briefly, $30 \mu \mathrm{L}$ of $1.8 \mathrm{mmol} / \mathrm{L}$ resazurin (Sigma, Steinheim, Germany) diluted in physiological saline was added to $3 \mathrm{~mL}$ of semen sample diluted 1: 2 with Beltsville thawing solution semen extender (Beltsville Thawing Solution, Truadeco, the Netherlands) and incubated at $37^{\circ} \mathrm{C}$ in a water bath for $10 \mathrm{~min}$. After incubation, two sub-samples of $1 \mathrm{~mL}$ were added to $1.5 \mathrm{~mL}$ of butanol (Merc, Germany). After rapid vortexing, samples were centrifuged at $3000 \times \mathrm{g}$ for $10 \mathrm{~min}$. Absorbance in the clear upper layer of butanol was measured at $610 \mathrm{~mm}$ (UV/VIS Spectrometer Lambda 12; Perkin Elmer Corp., Analytical Instruments, Norwalk, CT, USA). The within-run coefficient of variation, calculated as $7.79 \pm 4.06 \%$, confirmed satisfactory repeatability of the assay. Spearman rank correlation analysis was used to determine the correlation between resazurin reduction assay and semen parameters such as sperm density, morphology, motile sperm concentration, viable sperm concentration and sperm index. We observed the highest correlations of resazurin reduction with sperm concentration followed by motile sperm concentration and viable sperm concentration. Inverse correlations indicate that better values of seminal parameters are correlated with a lower level of absorbance, indicating a stronger reducing capacity of the dye (resazurin). There were correlations $(\mathrm{P}<0.001)$ between the reduction of resazurin and motile sperm concentration $(\mathrm{r}=0.81)$ and SI $(\mathrm{r}=0.82)$, therefore resazurin reduction assaay was further diagnostically evaluated according to motile sperm concentration and sperm index. Scatter-plots and linear regression equations are shown in Figures 7 and 8. 


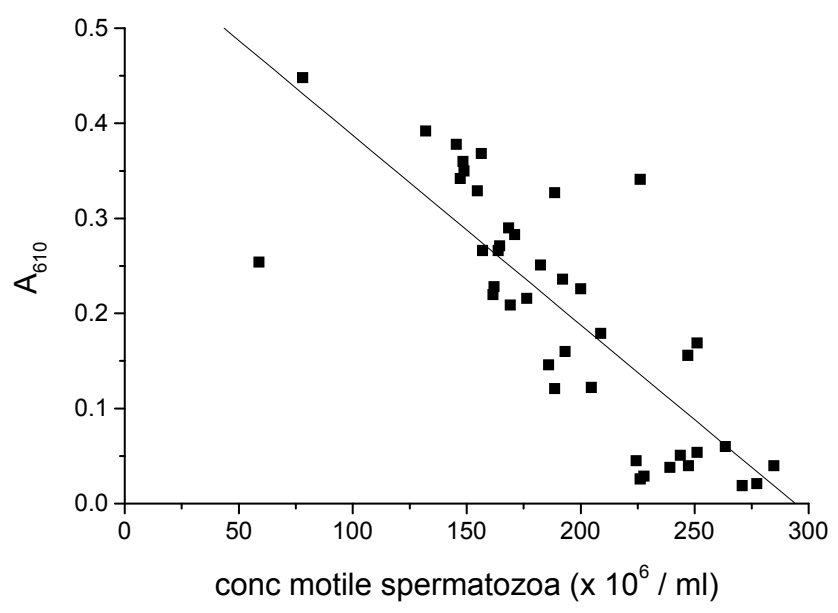

Fig. 7. Relationship between the reductions of resazurin expressed as absorbance and the concentration of motile spermatozoa Regression equation: $\mathrm{C}$ (motile spermatozoa) $=258.345-0.325 \times \mathrm{A}_{610}$

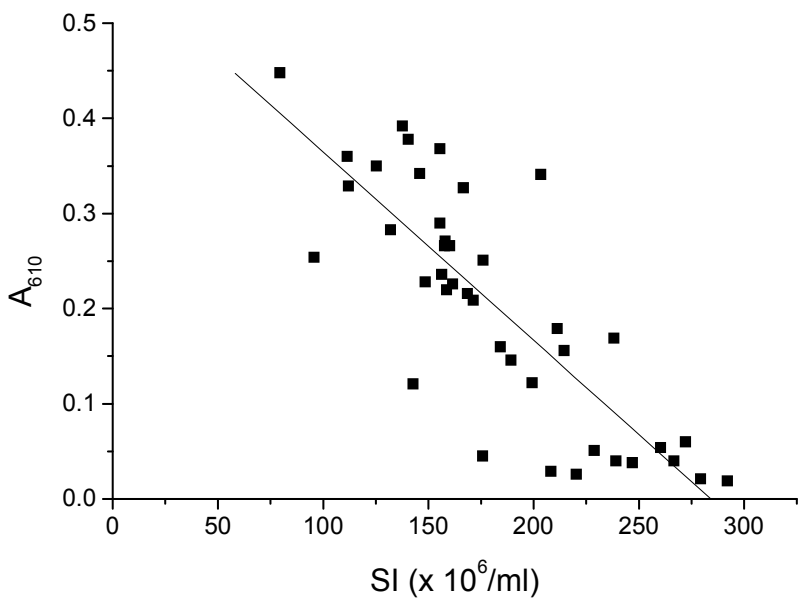

Fig. 8. Relationship between the reductions of resazurin expressed as absorbance and sperm index

Regression equation: $\mathrm{SI}=250.546-0.339 \times \mathrm{A}_{610}$

\subsection{Diagnostic evaluation of resazurin reduction assay}

Semen samples were divided into satisfactory (SAT) and unsatisfactory (UNSAT) according to various criteria. Criteria considering the concentration of motile sperm included preselected minimums of 160,200 and $240 \times 10^{6}$ sperm $/ \mathrm{mL}$. Criteria considering the concentration of motile, normal sperm (SI) included pre-selected minimums of 140, 180 and $220 \times 10^{6}$ sperm $/ \mathrm{mL}$. There was a significant difference between the absorbance values in 
groups of satisfactory and unsatisfactory semen samples $(\mathrm{P}<0.001)$ based on motile spermatozoa/mL and sperm index. Box plot in Fig.9 represents the values of $A_{610}$ in both groups divided according to motile sperm concentration and sperm index.

The performance of diagnostic tests is usually described in terms of sensitivity and specificity (Jones \& Payne, 1997). In the present study, receiver operating characteristics (ROC) analysis was used to determine the optimal cut-off value and diagnostic accuracy of the resazurin reduction assay by using boar semen. A complete picture of test accuracy is presented by the ROC plot, which provides a view of the whole spectrum of sensitivities (true positive rate) against one minus specificities (false positive rate) as functions of selected cut-off values (Greiner et al., 2000). A"good" test is one which has a high true positive rate and a low false positive rate and whose value, therefore, lies close to the top left-hand corner of the ROC curve (Petrie \& Watson, 1999). A global summary statistic of the diagnostic accuracy of the assay is quantified by the areas under ROC curves (AUC). Likelihood ratios (LR) are used to revise the probability of the semen status in individual samples (Greiner et al., 1995). However, a complete ROC analysis, including AUC, provides an index of accuracy by demonstrating the limits of a test's ability to discriminate between different semen status values (Zwieg et al., 1993).
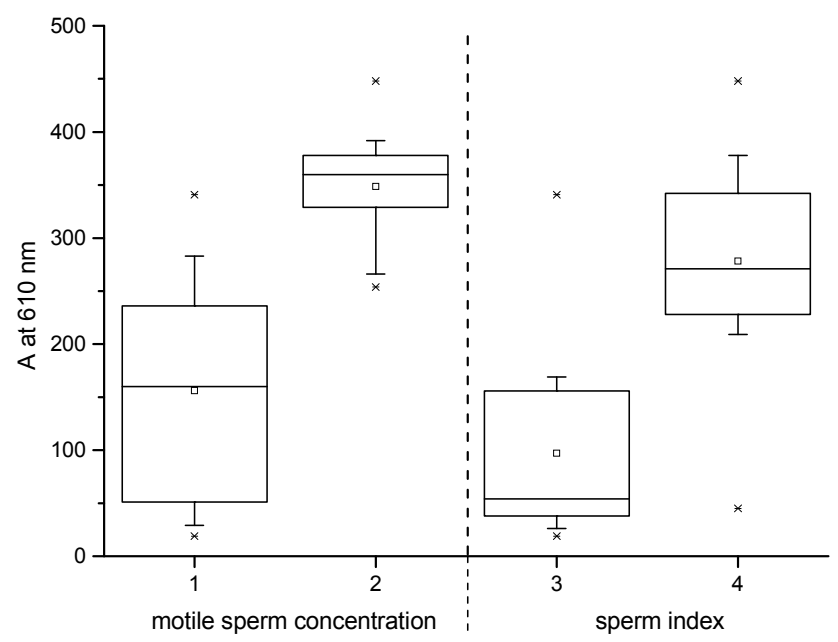

Fig. 9. Values of $A_{610}$ in groups of satisfactory (group 1, group 3) and unsatisfactory (group 2 , group 4) semen samples based on the motile sperm concentration $\left(160 \times 10^{6} \mathrm{sperm} / \mathrm{ml}\right)$ and sperm index $\left(180 \times 10^{6} \mathrm{sperm} / \mathrm{ml}\right)$

ROC curves (Analyse-it, General + Clinical Laboratory statistics, version 1.71; Analyse-it Software Ltd., Leeds, UK) were applied to identify optimal test cut-off values. A positive test result $(\mathrm{T}+)$ was recorded when spermatozoa in a sample reduced resazurin from blue to pink, resulting in $\mathrm{A}_{610}$ below the cut-off value. A negative test result (T-) was recorded when spermatozoa in a sample did not reduce resazurin from blue to pink, resulting in $\mathrm{A}_{610}$ above the cut-off value. Sensitivity (Se) and specificity (Sp) for each cut-off value were calculated as the proportion of positive test results $(\mathrm{T}+)$ for SAT samples and negative test results $\left(\mathrm{T}^{-}\right)$ for UNSAT samples according to complete $2 \times 2$ table (Table 1 ). 


$$
\begin{gathered}
\mathrm{Se}=\mathrm{TP} /(\mathrm{TP}+\mathrm{FN}) \\
\mathrm{Sp}=(\mathrm{TN}) /(\mathrm{TN}+\mathrm{FP})
\end{gathered}
$$

\begin{tabular}{|c|c|c|c|}
\hline \multirow{2}{*}{ Test result } & \multicolumn{2}{|c|}{ Semen samples status } & \multirow{2}{*}{ Total } \\
\cline { 2 - 3 } & Satisfactory (SAT) & $\begin{array}{c}\text { Unsatisfactory } \\
\text { (UNSAT) }\end{array}$ & \\
\hline Positive $(\mathrm{T}+)$ & True positive (TP) & False positive (FP) & TP + FP \\
\hline Negative $(\mathrm{T}-)$ & False negative (FN) & True negative (TN) & FN + TN \\
\hline Total & TP + FN & FP + TN & \\
\hline
\end{tabular}

Table 1. Complete $2 \times 2$ table

ROC curves plotted all sensitivity versus 1-specificity for the complete range of cut-off points (Greiner et al., 2000; Yuan et al., 2004). Sensitivity and specificity were estimated at 39 cut-off values. A diagonal line in a plot corresponds to a test that is positive or negative just by chance.

All possible combinations of sensitivity and specificity that can be achieved by changing the test's cut-off value were summarized by a single parameter; that is, AUC (Greiner et al., 2000). The slope of the ROC curve represents the LR for a diagnostic test, and the tangent at a point on the ROC curve corresponds to the LR for a single test value represented by that point (Choi et al., 1998).

$$
\mathrm{LR}=\mathrm{Se} /(1-\mathrm{Sp})
$$

The optimal cut-off values were selected based on the best balance of sensitivity, specificity and Youden index (J) along with larger increases in LR for each criterion value (Weiss et al., 2003-2004).

$$
\mathrm{J}=\mathrm{Se}+\mathrm{Sp}-1
$$

The diagnostic potential of resazurin reduction assay according to motile sperm concentration and SI was not different on the basis of AUC. The AUC was the same for criteria of $200 \times 10^{6}$ motile sperm $/ \mathrm{mL}$ and $180 \times 10^{6}$ motile, normal sperm $/ \mathrm{mL}$ (AUC=0.92; standard error for ROC curve $(\mathrm{SE})=0.047$ and 0.048 , respectively; $\mathrm{P}<0.0001$; Figure 10). On the basis of LR, absorbance lower than or equal to the optimal cut-off point were 11.3 and 7.1 times as likely to be found in satisfactory as in unsatisfactory semen samples according to SI and motile sperm concentration, respectively.

A plot of sensitivity, specificity and Youden index as a function of the cut-off value provides a useful visualisation and is helpful in selecting optimal cut-off values of the assay (Greiner, 2000). The selection of cut-off values of absorbance at $610 \mathrm{~nm}$ according to different criteria for motile sperm concentration and SI are presented in Figures 11 and 12, respecitvely.

Values of Youden index peaked at a cut-off point of $\mathrm{A}_{610}$ at 0.209 for pre-selected minimum concentration of motile sperm concentration of $200 \times 10^{6}$ sperm $/ \mathrm{mL}$ (Figure 12B) and SI of $180 \times 10^{6}$ sperm $/ \mathrm{mL}$ (Figure 12B). The optimal cut-off value at $\mathrm{A}_{610}$ of 0.209 therefore provided the best discrimination power according to both motile sperm concentration and SI. At this point, maximum overall accuracy was achieved for both cases. This cut-off value yielded estimates of sensitivity of $88.2 \%$ and $94.1 \%$ with corresponding specificities of $87.5 \%$ and $91.7 \%$ for motile sperm concentration and SI, respectively. 
A

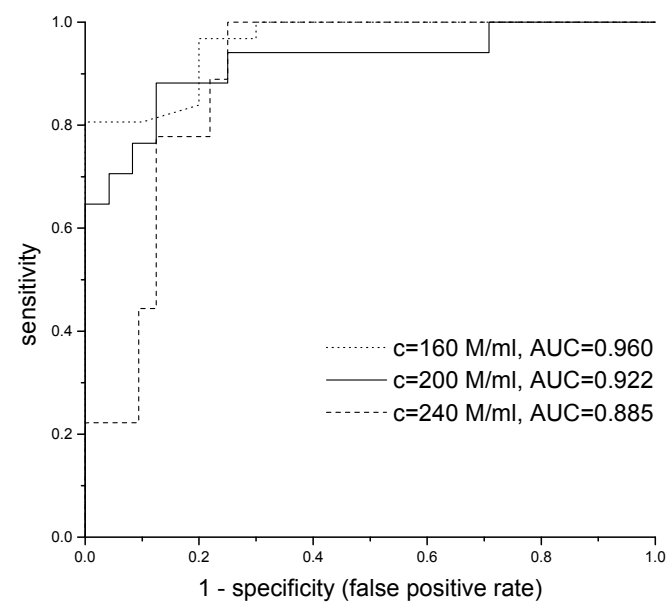

B

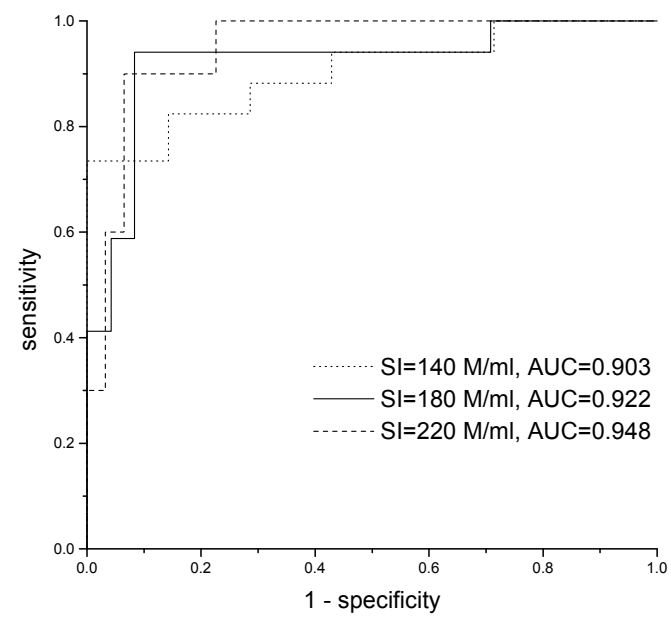

Fig. 10 (A, B). ROC plots of resazurin reduction assay for identifying semen samples with pre-selected minimum concentration of motile sperm concentration (A) and motile and normal sperm (B).

However, in clinical use of the test, it is often important to $100 \%$ correctly identify satisfactory or unsatisfactory samples. Therefore, a cut-off value of $\mathrm{A}_{610}$ at 0.342 was selected to enable $100 \%$ correct identification of unsatisfactory semen samples. For both criteria, the test is $100 \%$ sensitive at $A_{610}$ of 0.342 . A cut-off value at $A_{610}$ of 0.121 gives $100 \%$ specificity for motile sperm concentration and $95.8 \%$ specificity for SI. For pre-selected minimum concentration of motile sperm concentration of $160 \times 10^{6}$ sperm $/ \mathrm{mL}$ and SI of $140 \times 10^{6}$ sperm $/ \mathrm{mL}, 100 \%$ specificity was obtained at the optimal cut-off value of $\mathrm{A}_{610}$ at 0.254 , whereas only moderate levels of sensitivity were observed $(80.6 \%$ and $73.5 \%$, respectively; Figures $11 \mathrm{~A}$ and $12 \mathrm{~A}$ ). In contrast, at the highest criteria values $100 \%$ sensitivity corresponded to only moderate levels of specificity (Figures 11C and 12C). In contrast, semen samples with $\mathrm{A}_{610}$ below 0.121 in the resazurin reduction assay were $100 \%$ 
A

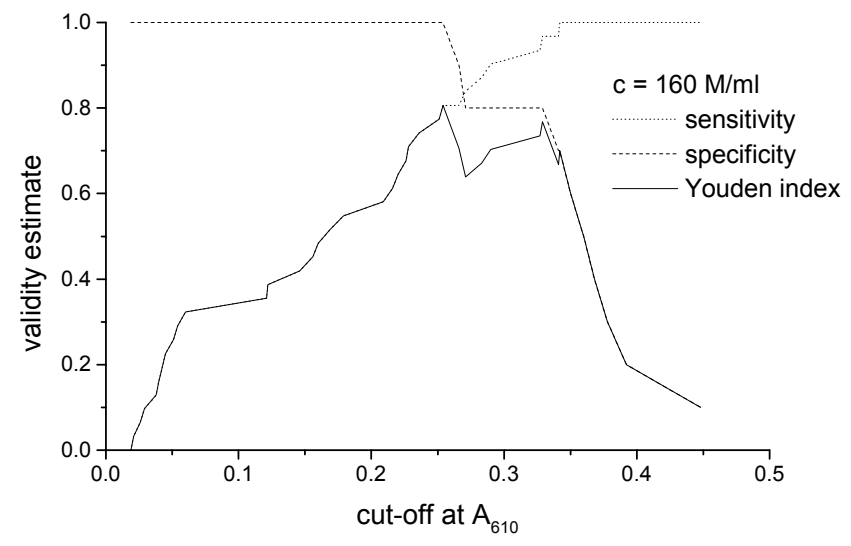

B

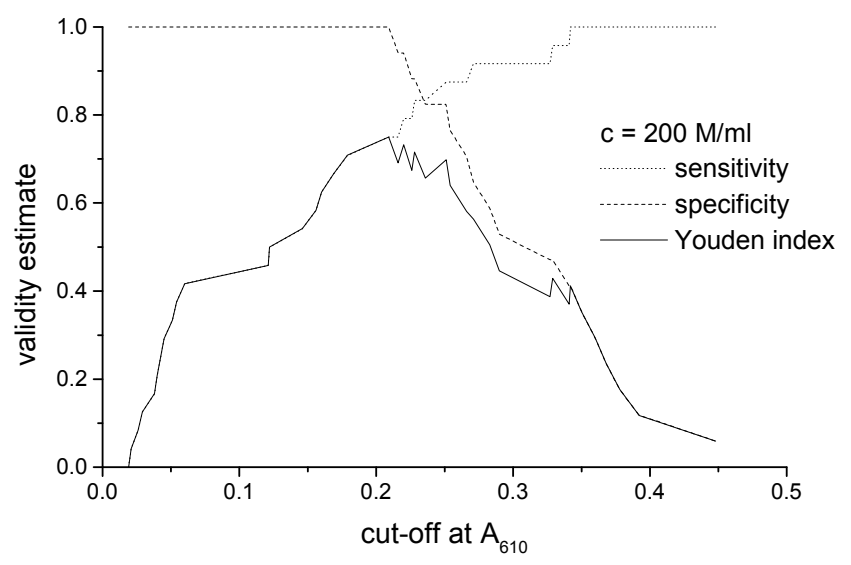

C

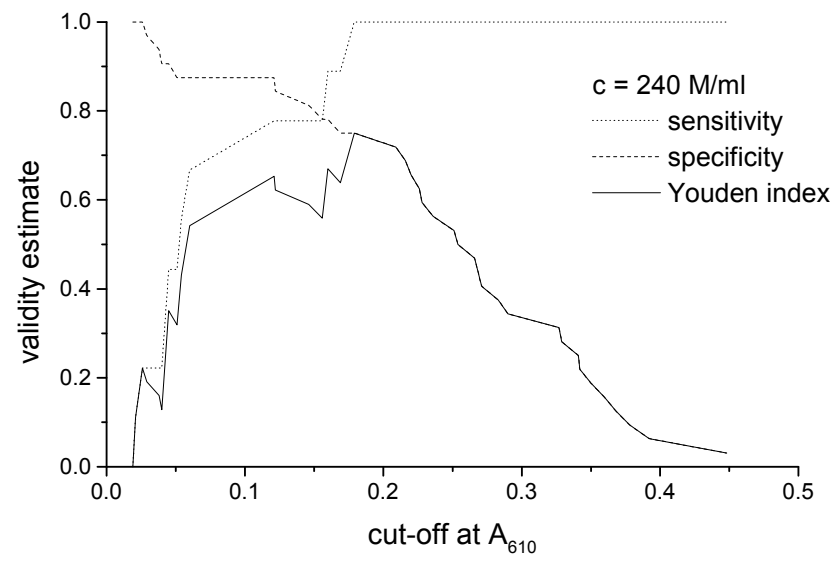

Fig. 11 (A, B, C). Plot of the diagnostic specificity, sensitivity and Youden index of resazurin reduction assay according to motile sperm concentration as a function of the cut-off value at $610 \mathrm{~nm}$. 

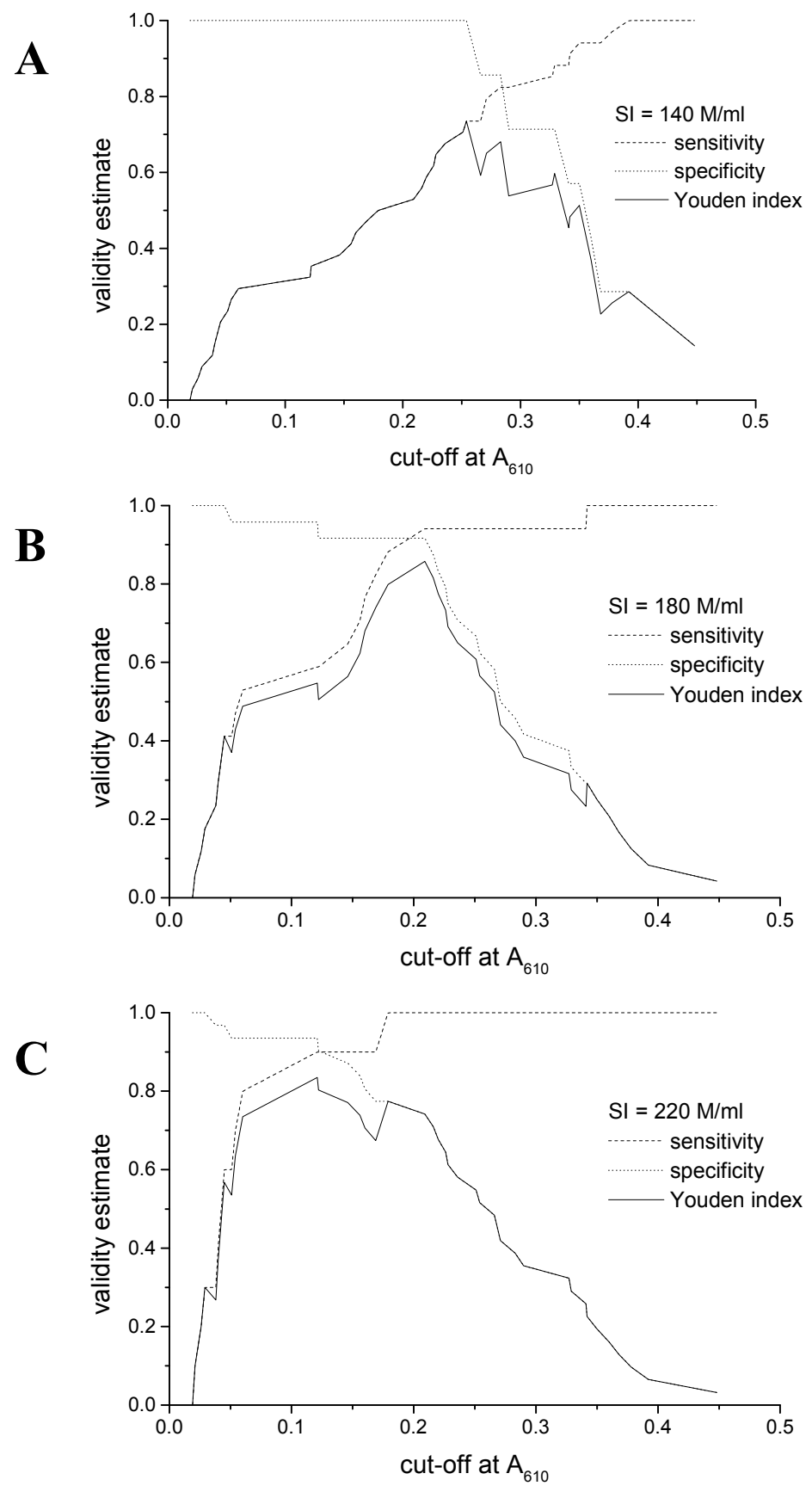

Fig. 12 (A, B, C). Plot of the diagnostic specificity, sensitivity and Youden index of resazurin reduction assay according to sperm index as a function of the cut-off value at $610 \mathrm{~nm}$. 
and $95.8 \%$ correctly identified as satisfactory according to the criteria of $200 \times 10^{6}$ motile sperm/mL or $180 \times 10^{6}$ motile, normal sperm/mL, respectively. In our quantitative test, the maximum overall accuracy of $92.9 \%$ confirmed the high discrimination power for boar semen according to a criterion value of SI at $180 \times 10^{6}$ sperm $/ \mathrm{mL}$.

\subsection{Stability of butanol extracts in terms of $\mathbf{A 6 1 0}$}

After developing the assay, we wondered if it was possible to measure the absorbance at a later date, i.e. within a day or even a week of the assay. A satisfactory level of agreement would indicate that the modification was successful, which in turn would greatly enhance the usefulness of the assay as it could then be performed even if a spectrophotometer was not immediately available.

We measured the $A_{610}$ of each butanol extract of 112 samples on days 0, 1 and 7 after storage at $4^{\circ} \mathrm{C}$. The differences were obtained between $\mathrm{A}_{610}$ at day 0 (A0) and day 1 (A1) and between days 0 (A0) and 7 (A7).

The limits of agreement were calculated as follows: limits $=\bar{d} \pm 2$ sdiff, where $\bar{d}$ is the mean of differences for all the samples, and sdiff is the standard deviation of the differences. 2sdiff is also named the reproducibility coefficient. Differences between absorbances (A1 A0) were plotted against their average value $((\mathrm{A} 1+\mathrm{A} 0) / 2)$ for each sample. Satisfactory agreement is achieved when minimum $95 \%$ of the absolute differences are less than the reproducibility coefficients (Petrie \& Watson, 1999).

It is necessary to establish that a method is repeatable before comparing two measurements for reproducibility (Petrie \& Watson, 1999). The within-run coefficient of variation, calculated as $7.79 \pm 4.06 \%$, confirmed satisfactory repeatability of the method, therefore the pairs of measurement of A610 were allowed to compare. The differences between measurements $\left(\mathrm{A}_{610}\right)$ immediately after centrifugation (day 0$)$ and after 7 days were plotted against the average of these values. $95.54 \%$ of differences lie within the limits of agreement (Fig. 13).

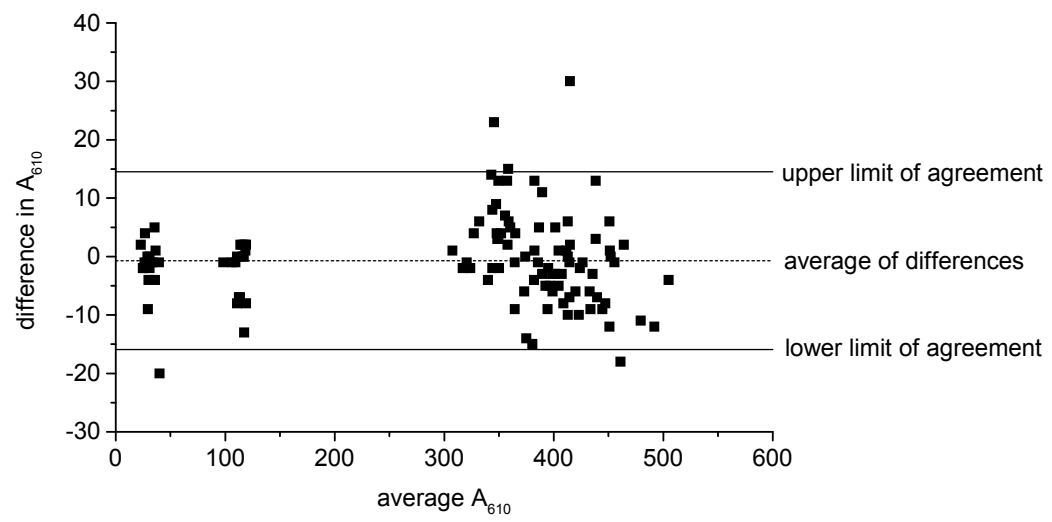

Fig. 13. $\mathrm{A}_{610}$ values as a function of time of measurement

Measurements obtained on the day of performing the test and the measurements after 24 hours also agree; $99.1 \%$ of the differences lie within the limits of agreement (data not shown). The results obtained leading to the conclusion that we can measure $\mathrm{A}_{610}$ of butanol 
extracts within 7 days from the day of test performing, confirming a great practical value of the method.

In a diagram of the differences between absorbances plotted against their average, the scatter of the points is random (Fig. 13) indicating, that the size of the discrepancy between the two absorbance is not related to the size of the absorbance. More than $95 \%$ of absolute differences were less than the reproducibility coefficients in both cases of testing the stability of butanol extracts. This is a satisfactory agreement, therefore we can measure the absorbance immediately after performing the test or within 7 days of that time. Therefore the test is useful even if spectrophotometer is not available at the location of semen evaluation. The results obtained leading to the conclusion that we can measure $\mathrm{A}_{610}$ of butanol extracts within 7 days from the day of test performing, confirming a great practical value of the method.

\section{Conclusions}

The usefulness of sperm counting is greatly enhanced by the simplicity of determination by photometer (Photometer SDM5, MiniTüb, Germany) in on-farm AI laboratories. The use of photometer for determining sperm concentration would, therefore, be of benefit also to livestock producers in evaluating the quality of boar semen.

The resazurin reduction assay was shown to be a reliable, easy-to-perform test that requires no sophisticated equipment. It was demonstrated that the results of the assay can be used to select semen samples with minimum requirements of sperm concentration, motility and normal morphology, which are all combined in sperm index. Because reproductive performance depends on metabolic processes, the assessment of metabolic rates of spermatozoa could provide even better or more complete information about semen quality than other tests. It allows the concentration of active spermatozoa to be determined, and may provide a better means of evaluating semen quality than assessing the characteristics, mentioned above, independently. Expressing the latter in semen evaluation is complex, although fertility results from insemination with evaluated semen could provide a gold standard of fertilizing capacity. Additional research is required for relevant and valid information about replacing or updating the methodology of semen evaluation.

\section{Acknowledgements}

This work was supported by the Slovenian Ministry of Higher Education, Science and Technology, programme group "Endocrine, immune, nervous and enzyme responses in healthy and sick animals" (P4-0053).

Special thanks go to author's collegues who contributed to the research work, presented in this chapter: Janko Mrkun, DVM, PhD, Marjan Kosec, DVM, PhD, Janez Kunc, DVM, MSc, Maja Zakošek Pipan, DVM.

\section{References}

Anzar, M., Kroetsch, T., Buhr, M.M. (2009). Comparison of different method for assessment of sperm concentration and membrane integrity with bull semen. J Androl, 30, 6, pp. (661-668) 
Bland J.M. \& Altman D.G. (1999). Measuring agreement in method comparision studies. Stat Methods Med Res 8, pp. (135-160)

Brazil, C., Swan, S.H., Tollner, C.R., Treece, C., Drobnis, E.Z., Wang, C., Redmon, J.B. \& Overstreet, J.W. (2004). Quality control of laboratory methods for semen evaluaion in a multicenter research study. J. Androl., 25, pp. (645-656)

Carter, R.A., Ericsson, S.A., Corn, C.D., Weyerts, P.R., Dart, M.G., Escue, S.G. \& Mesta J. (1998). Assessing the fertility potential of equine semen samples using the reducible dyes methylene green and resazurin. Arch Andro, 40, 1, pp. (59-66)

Camus, A., Camugli, S., Leveque, C., Schmitt, E. \& Staub, C. (2011). Is photomertry an accurate and reliable method to assess boar semen concentration? Theriogenology, 75, pp. (577-583)

Choi, B.C. (1998). Slopes of a Receiver Operating Characteristic Curve and Likelihood Ratios for a Diagnostic Test. Am J Epidemiol, 148, pp. (1127-1132)

Christensen, P., Stenvang, J. \& Godfrey, W. (2004) A flow cytometric method for rapid determination of sperm concentration and viability in mammalian and avian semen. J Androl, 25, 2, pp. (255-264)

Christensen, P., Stryhn, H. \& Hansen, C. (2005). Discrepancies in the determination of sperm concentration using Bürker-Türk, Thoma and Makler counting chambers. Theriogenology, 63, 4, pp. (992-1003)

Cooper, T.A., Wang, S., Liu, Y., Bunch, T.D. \& Holyoak, G.R. (1996). A new method to evaluate the viability of cryopreserved ram semen using a resazurin reduction assay. Theriogenology, 45, 1, pp. 313.

Dart, M.G., Mesta, J., Creshaw, C. \& Ericsson, S.A. (1994). Modified resazurin reduction test for determining the fertility potential of bovine spermatozoa. Arch Androl, 33, pp. (71-75)

Fuse, H., Okumura, M., Kazama, T. \& Katayama T. (1993). Comparison of resazurin test results with various sperm parameters. Andrologia, 25, pp, (153-157)

Glass, R.H., Drouin, M.T., Ericsson, S.A., Marcoux, L.J., Ericsson, R.J. \& Sullivan H. (1991). The resazurin reduction test provides an assessment of sperm activity. Fertil Steril, 56, pp. (43-46)

Greiner, M., Sohr, D.\& Göbel, P. (1995). A modified ROC analysis for the selection of cut-off values and the definition of intermediate results of serodiagnostic tests. J Immunol Methods, 185, pp. (123-132)

Greiner, M., Pfeiffer, D. \& Smith, R.D. (2000). Principles and practical application of the receiver-operating characteristic analysis for diagnostic tests. Prev Vet Med, 54, pp. $(23-41)$

Hafez, E.S.E. (1993). Semen evaluation. In: Reproduction in Farm animals (6 ${ }^{\text {th }}$ ed.). Hafez, E.S.E., pp. (405-423), Philadelphia, PA

Hansen, C., Vermeiden, T., Vermeiden, J.P.W., Simmet, C., Day, B.C. \& Feitsma, H. (2006). Comparision of FACSCount AF system, improved Neubauer hemacytometer, Corning 254 photometer, SpermVision, UltiMate and NucleoCounter SP-100 for determination of sperm concentration of boar semen. Theriogenology, 66, pp. (21882194)

Holt, W.V. \& Medrano A. (1997). Assessment of boar sperm function in relation to freezing and storage. J Reprod Fertil, Suppl., 52, pp. (213-222)

Imade, G.E., Towobola, O.A., Sagay, A.S. \& Otubu, J.A. (1993). Discrepancies in sperm count using improved Neubauer, Makler, and Horwells counting chambers Arch Androl, 31,1 , pp. $(17-22)$ 
Jensen, A.L. \& Kjelgaard-Hansen, M. (2006). Method comparison in the clinical laboratory, Vet Clin Pathol., 35, pp. (276-286)

Johnson, L.A., Weitze, K.F., Fiser, P. \& Maxwell, W.M. (2000). Storage of boar semen. Anim Reprod Sci, 62, pp. (143-172)

Jones, R.G. \& Payne, R.B., (1997). Analytical methods: control and comparison. In: Clinical Investigation and Statistics in Laboratory Medicine, Jones, R.G. \& Payne, R.B., pp. (2765), ACB Venture Publications, London

Juonala, T., Lintukangas, S., Nurttila, T. \& Andresson, M. (1998). Relationship between semen quality and fertility in 106 AI-Boars, Reprod Domest Anim, 33, pp. (155-158)

Keel, B., Quinn, P., Schmidt, C., Serafy, N, Jr, Serafy, N. Sr. \& Schalue, T. (2000). Results of the American association of Bioanalysts national proficiency testing programme in andrology, Hum Reprod, 15, pp (680-686)

Knuth, U.A., Neuwinger, J. \& Nieschlag, E. (1989). Bias to routine semen analysis by uncontrolled changes in laboratory environment - detection by long-term sampling of monthly means for quality control, Int J Androl, 12, pp. (375-383)

Liu, D.Y. \& Baker, H.W.G. (2002). Evaluation and assessment of semen for IVF/ICSI, Asian J Androl, 4, pp. (281-285)

Ma, D. \& Smith, F.G. (2003). Correlation and regression. In: Key topics in Clinical Research, Smith, F.G. \& Smith, J.R., pp. (147-151), BIOS Scientific Publishers Limited, Oxford

Maatson, P. (1995). External quality assessment for semen analysis and sperm antibody detection: results of a pilot scheme, Hum Reprod, 10, pp. (620-625).

Mahmoud, A.M., Comhaire, F.H., Vermeulen, L. \& Andreou, E. (1994). Comparison of the resazurin test, adenosine triphosphate in semen, and various sperm parameters, Hum Reprod, 9, pp. (1688-1693)

Mahmoud, A.M.A., Depoorter, B., Piens, N. \& Comhaire, F.H., (1997). The performance of 10 different methods for the estimation of sperm concentration, Fertil and Steril, 68, 2, pp. (340-345)

Martin, L.M., Crenshaw, C.C., Dean, J.A. Jr., Dart, M.G., Purdy, P.H. \& Ericsson, S.A. (1999). Determination of the number of motile sperm within an ovine semen sample using resazurin, Small Rumin Res, 32, pp. (161-165)

Mesta, J., Ericsson, S.A., Dart, M.G., Wansley, R.G. \& Weyerts, P.R. (1995) Assesment of fertility potential of porcine spermatozoa using the reducible dyes methylene green and resazurin. Journal of Animal Science, 73, (Suppl. 1), pp. (26)

Mrkun, J., Kosec, M., Zakošek, M., Zrimšek, P. (2007). Method agreement between measuring of boar sperm concentration using makler chamber and photometer. Acta vet (Beogr.), 57, 5/6, pp. (563-572)

Neuwinger, J., Behre, H.M. \& Nieschlag, E. (1990). External quality control in the andrology laboratory: an experimental multicenter trial, Fertil Steril, 54, pp. (308-314)

Petrunkina, A.M., Waberski, D., Günzel-Apel, A.R. \& Töpfer-Petersen, E. (2007). Determinants of sperm quality and fertility in domestic species. Reproduction, 134, pp. (3-17)

Petrie, A. \& Watson, P. (1999). Additional Topics. In: Statistics for veterinary and animal science, Petrie, A., Watson, P., pp. (168-181), Blackwell Science, Oxford

Prathalingam, N., Holt, W., Revell, S., Jones, S. \& Watson, P. (2006). The precision and accuracy of six different methods to determine sperm concentration. J Androl, 27, 2, pp. (257-262)

Quintero-Moreno, A., Rigau, T. \& Rodriguez-Gil, J.E. (2004). Regression analysis and motile sperm subpopulation structure study as improving tools in boar semen quality analysis, Theriogenology, 61, pp. (673-690) 
Rahman, N.A. \& Kula, K. (1997). Enlarged spectrum of seminological diagnoses using the resazurin colour reaction, a spectrophotometric application, Int J Andro, 20, pp. (1722)

Reddy Venkata Rami, K., Meherji, P.K., Gokral, J.S. \& Shahani, S.K. (1997). Resazurin reduction test to evaluate semen quality. Indian Journal of Experimental Biology, 35, pp. (369-373)

Reddy Venkata Rami \& K., Bordekar, A.D. (1999). Spectrophotometric analysis of resazurin reduction test and semen quality in men. Indian J Exp Biol, 37, pp. (782-786)

Sokol, R.Z., Shulman, P. \& Paulson, R.J. (2000). Comparison of two methods for the measurement of sperm concentration. Fertil Steril, 73, 3, pp. (591-594)

Tejerina, F., Buranaamnuay, K., Saravia, F,, Wallgren, M. \& Rodriguez-Martinez, H. (2008). Assessment of motility ejaculated, liquid-stored boar spermatozoa using computerized instruments, Theriogenology, 69, pp. (1129-1138)

Tsakmakidis, I.A., Lymberopoulos, A.G. \& Khalifa, T.A.A. (2010). Relationship between sperm quality traits and filed-fertility of porcine semen. J Vet Sci, 11, 2, pp. (151-154)

Twormey, P. (2004). Plasma glucose measurement with the Yellow Springs Glucose 2300 STAT and the Olympus AU640. J Clin Pathol, 57, pp. (752-754).

Twormey, P. (2005). How do we really compare methods in the clinical laboratory? Proceedings of Statistics Workshop \& Clinics, EuroMedLab, Glasgow, May 2005

Vyt, P., Maes, D., Rijsselaere, T., Dejonckheere, E., Castryck, F. \& Van Soom, A. (2004). Motility Assessment of Porcine Spermatozoa: a Comparison of Methods. Reprod Dom Anim, 30, pp. (447-453)

Yuan, Y.M., Xin, Z.C., Jiang, H., Guo, Y.J., Liu, W.J., Tian, L. \& Zhu, J.C. (2004). Sexual function of premature ejaculation patients assayed with Chinese Index of Premature Ejaculation, Asian J Androl, 6, pp. (121-126)

Waberski, D., Schapmann, E., Henning, H., Reisenbeck, A. \& Brandt, H. (2011). Sperm chromatin structural integrity in normospermic boars is not related to semen storage and fertility after routine AI, Theriogenology, 75, pp. (337-345)

Wang, S., Holyoak G.R., Panter, K.E., Liu, G., Evans, R.C., Bunch, T.D. (1998) Resazurin reduction assay for ram sperm metabolic activity measured by spectrophotometry. Proc Soc Exp Biol Med, 217, pp. (197-202)

Weiss, H.L., Niwas, S., Grizzle, W.E. \& Piyathilake, C. (2003-2004). Receiver operating characteritic (ROC) to determine cut-off points of biomarkers in lung cancer patients. Dis Markers, 19, pp. (273-278)

Woelders, H. (1991). Overview of in vitro methods for evaluation of semen quality, Reprod Domest Anim Suppl, pp. (145-164)

Zalata, A.A., Lammertijn, N., Christoper, A. \& Comhaire, F.H. (1998). The correlates and alleged biochemical background of the resazurin reduction test in semen. Int $J$ Androl, 21, pp. (289-294)

Zwieg, M.H. \& Campbell, G. (1993). Receiver-Operating Characteristic (ROC) Plots: A Fundamental Evaluation Tool in Clinical Medicine, Clin. Chem., 39/40, pp. (561-577)

Zrimšek, P., Kunc, J., Kosec, M., Mrkun, J. (2004). Spectrophotometric application of resazurin reduction assay to evaluate boar semen quality. Int J Androl, 27, 1, pp. (57-62)

Zrimšek, P., Kosec, M., Kunc, J., Mrkun, J. (2006). Determination of the diagnostic value of the resazurin reduction assay for evaluating boar semen by receiver operating characteristic analysis. Asian J Androl, 8, 3, pp. (343-348) 


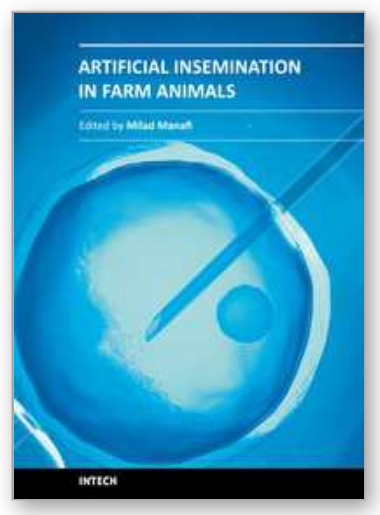

\author{
Artificial Insemination in Farm Animals \\ Edited by Dr. Milad Manafi
}

ISBN 978-953-307-312-5

Hard cover, 300 pages

Publisher InTech

Published online 21, June, 2011

Published in print edition June, 2011

Artificial insemination is used instead of natural mating for reproduction purposes and its chief priority is that the desirable characteristics of a bull or other male livestock animal can be passed on more quickly and to more progeny than if that animal is mated with females in a natural fashion. This book contains under one cover 16 chapters of concise, up-to-date information on artificial insemination in buffalos, ewes, pigs, swine, sheep, goats, pigs and dogs. Cryopreservation effect on sperm quality and fertility, new method and diagnostic test in semen analysis, management factors affecting fertility after cervical insemination, factors of noninfectious nature affecting the fertility, fatty acids effects on reproductive performance of ruminants, particularities of bovine artificial insemination, sperm preparation techniques and reproductive endocrinology diseases are described. This book will explain the advantages and disadvantages of using Al, the various methodologies used in different species, and how Al can be used to improve reproductive efficiency in farm animals.

\title{
How to reference
}

In order to correctly reference this scholarly work, feel free to copy and paste the following:

Petra Zrimšek (2011). Evaluation of a New Method and Diagnostic Test in Semen Analysis, Artificial Insemination in Farm Animals, Dr. Milad Manafi (Ed.), ISBN: 978-953-307-312-5, InTech, Available from: http://www.intechopen.com/books/artificial-insemination-in-farm-animals/evaluation-of-a-new-method-anddiagnostic-test-in-semen-analysis

\section{INTECH}

open science | open minds

\section{InTech Europe}

University Campus STeP Ri

Slavka Krautzeka 83/A

51000 Rijeka, Croatia

Phone: +385 (51) 770447

Fax: +385 (51) 686166

www.intechopen.com

\section{InTech China}

Unit 405, Office Block, Hotel Equatorial Shanghai

No.65, Yan An Road (West), Shanghai, 200040, China

中国上海市延安西路 65 号上海国际贵都大饭店办公楼 405 单元

Phone: +86-21-62489820

Fax: $+86-21-62489821$ 
(C) 2011 The Author(s). Licensee IntechOpen. This chapter is distributed under the terms of the Creative Commons Attribution-NonCommercialShareAlike-3.0 License, which permits use, distribution and reproduction for non-commercial purposes, provided the original is properly cited and derivative works building on this content are distributed under the same license. 